\title{
Optimal Allocation of Smart Substations in a Distribution System Considering Interruption Costs of Customers
}

Sun, Lei; You, Shi; Hu, Junjie; Wen, Fushuan

Published in:

IEEE Transactions on Smart Grid

Link to article, DOI:

10.1109/TSG.2016.2642163

Publication date:

2018

Document Version

Peer reviewed version

Link back to DTU Orbit

Citation $(A P A)$ :

Sun, L., You, S., Hu, J., \& Wen, F. (2018). Optimal Allocation of Smart Substations in a Distribution System Considering Interruption Costs of Customers. IEEE Transactions on Smart Grid, 9(4), 3773-3782.

https://doi.org/10.1109/TSG.2016.2642163

\section{General rights}

Copyright and moral rights for the publications made accessible in the public portal are retained by the authors and/or other copyright owners and it is a condition of accessing publications that users recognise and abide by the legal requirements associated with these rights.

- Users may download and print one copy of any publication from the public portal for the purpose of private study or research.

- You may not further distribute the material or use it for any profit-making activity or commercial gain

- You may freely distribute the URL identifying the publication in the public portal 


\title{
Optimal Allocation of Smart Substations in a Distribution System Considering Interruption Costs of Customers
}

\author{
Lei Sun, Shi You, Member, IEEE, Junjie Hu, Member, IEEE, Fushuan Wen
}

\begin{abstract}
One of the major functions of a smart substation (SS) is to restore power supply to interrupted customers as quickly as possible after an outage. The high cost of a smart substation limits its widespread utilization. In this paper, a smart substation allocation model (SSAM) to determine the optimal number and allocation of smart substations in a given distribution system is presented, with the upgrade costs of substations and the interruption costs of customers taken into account. Besides, the reliability criterion is also properly considered in the model. By linearization strategies, the SSAM is simplified into a mixed integer linear programming problem which could be solved efficiently with commercial solvers. Finally, the performance of the proposed methodology is demonstrated by the standard RBTS-BUS 4 test system and a medium voltage power distribution system in Denmark.
\end{abstract}

Index Terms - distribution system, smart substation allocation, upgrade cost, interruption cost, reliability cost, service restoration, outage management.

\section{NOMENCLATURES}

A. Indices and Sets:
$f, S_{f} \quad$ Index and set of feeders.
$l, S_{l} \quad$ Index and set of line segments.
$s, S_{s} \quad$ Index and set of $10 / 0.4 \mathrm{kV}$ substations.
$k, S_{k} \quad$ Index and set of types of customers.
$w, S^{\mathrm{TS}} \quad$ Index and set of tie switches.
$z, S_{z} \quad$ Index and set of sections.
$S_{w}^{S T S}$
Set of substations which are located on all sides
$S_{w}^{T F S} \quad$ Set of substations which are located between of a tie switch $w$. substation $s\left(s \in S_{w}^{S T S}\right)$ and the feeder supplying power to substation $s$.

Manuscript received on July 9, 2016. This work is jointly supported by National Natural Science Foundation of China (51477151), and Specialized Research Fund for the Doctoral Program of Higher Education of China (20120101110112).

L. Sun and F. Wen are both with the College of Electrical Engineering, Zhejiang University, Hangzhou 310027, China (Emails: sunleieee@ gmail.com fushuan.wen@gmail.com). F. Wen is also with Universiti Teknologi Brunei (UTB), Bandar Seri Begawan, Brunei (Email: fushuan.wen@utb.edu.bn).

S. You and J. Hu are with the Center for Electric Power and Energy, Department of Electrical Engineering, Technical University of Denmark, 2800 Kgs. Lyngby, Denmark (Emails: sy@elektro.dtu.dk; junhu@elektro.dtu.dk).
$\Omega_{f} \quad$ Set of line segments and substations supplied by feeder $f$.

$\Omega_{f, z} \quad$ Set of line segments and substations within section $z$ supplied by feeder $f$.

$\Omega_{f, z}^{\text {up }} \quad$ Set of substations in the upstream of section $z$ supplied by feeder $f$.

$\Omega_{f, z}^{\text {down }} \quad$ Set of substations in the downstream of section $z$ supplied by feeder $f$.

$\Omega_{f, l, s}^{S S} \quad$ Set of substations between line $l$ and substation $s$ supplied by feeder $f$.

B. Parameters

$S_{\text {AIDI }}^{\lim } \quad$ Threshold of the allowed SAIDI value.

$\lambda_{f, l} \quad$ Probability of a fault occurred on line segment $l$ at feeder $f$.

$p_{f, s, k} \quad$ Power consumption of type $k$ at substation $s$ supplied by feeder $f$.

$C_{f, s}^{C I C} \quad$ Capital investment cost of upgrading substation $s$ supplied by feeder $f$.

$C_{f, s}^{I C} \quad$ Installment cost of upgrading substation $s$ at feeder $f$.

$C_{f, s}^{M C} \quad$ Maintenance and operation cost of upgrading substation $s$ at feeder $f$.

$N_{f, s} \quad$ Number of customers supplied by substation $s$ at feeder $f$.

$t_{s, f, l}^{\mathrm{cur}} \quad$ Failure duration of substation $s$ when a fault occurs on line $l$ of feeder $f$ in the current distribution system.

$t_{s, f, l}^{\text {smart }} \quad$ Failure duration of substation $s$ when a fault occurs on line $l$ of feeder $f$ if the fault can be isolated by a smart substation between substation $s$ and line $l$.

$t_{z}^{\text {uss }} \quad$ Time spent on opening the closest upstream switch in section $z$ manually.

$t_{z}^{\mathrm{dts}} \quad$ Time spent on opening the closest downstream switch in section $z$ and closing a tie switch manually.

$t_{z}^{\text {uss } \_ \text {a }} \quad$ Time spent on opening an upstream switch in section $z$ automatically. 


\begin{tabular}{|c|c|}
\hline$t_{z}^{\mathrm{dts} \_\mathrm{a}}$ & $\begin{array}{l}\text { Time spent on opening the closest downstream } \\
\text { switch in section } z \text { and closing a tie switch } \\
\text { automatically. }\end{array}$ \\
\hline$N_{g}$ & $\begin{array}{l}\text { Number of separating points in the outage cost } \\
\text { function curve of customers. }\end{array}$ \\
\hline$t_{g}, t_{g+1}$ & $\begin{array}{l}\text { Time points of separating segment } g \text { in the } \\
\text { interruption cost curve of customers where } \\
g=1, \ldots, N_{g}-1 \text {. }\end{array}$ \\
\hline$a_{g}, b_{g}$ & $\begin{array}{l}\text { Slope and constant term of each segment } g \text { in } \\
\text { the piecewise interruption cost function of } \\
\text { customers where } g=1,2, \ldots, N_{g}-1 \text {. }\end{array}$ \\
\hline$\Upsilon_{z}$ & Vector of failure probabilities of lines in section \\
\hline & $z$ \\
\hline $\bar{\Upsilon}_{z, l}$ & Vector of failure location in line $l$ of section $z$ \\
\hline & $\begin{array}{l}\text { in which only the element corresponding to line } \\
l \text { is } 1 \text { and any other element is } 0 .\end{array}$ \\
\hline $\mathbf{T}_{z}^{d t}$ & $\begin{array}{l}\text { Time vector spent on detecting the candidate } \\
\text { faulty lines in section } z \text {. }\end{array}$ \\
\hline $\mathbf{T}_{z}^{r t}$ & $\begin{array}{l}\text { Time vector spent on repairing the candidate } \\
\text { faulty lines in section } z \text {. }\end{array}$ \\
\hline $\mathbf{T}_{z}^{a t}$ & $\begin{array}{l}\text { Time vector spent on restoring power supply to } \\
\text { the faulty section } z \text { after all faulty lines are } \\
\text { repaired. }\end{array}$ \\
\hline$p_{z}$ & Power consumption in section $z$. \\
\hline$T_{z}$ & $\begin{array}{l}\text { Maximum number of time slots needed to } \\
\text { restore the faulty section } z \text {. }\end{array}$ \\
\hline $\mathbf{G}_{z}$ & $\begin{array}{l}\text { Ancillary matrix with all upper triangular } \\
\text { elements to be } 1 \text { and all lower triangular } \\
\text { elements to be } 0 \text {. }\end{array}$ \\
\hline$t_{k, g}^{a d d}$ & $\begin{array}{l}\text { The } g^{\text {th }} \text { separation point of the piecewise } \\
\text { interruption cost function of customers with } \\
\text { type } k \text {. }\end{array}$ \\
\hline$y_{k, g}$ & $\begin{array}{l}\text { Cost of customers of type } k \text { with the outage } \\
\text { duration } t_{k, g}^{\text {add }} \text {. }\end{array}$ \\
\hline 0 & A very small number. \\
\hline
\end{tabular}

C. Functions

$S_{A I D I}(\mathbf{X}) \quad$ Value of the SAIDI index with the allocations of smart substations as inputs.

$E_{z}^{E N S} \quad$ Expected Energy Not Supplied (EENS) for any section $z$ when a faulty line exists in section $z$.

$f_{k}(t) \quad$ Interruption cost of customers of type $k$ within interruption time $t$.

$C^{\mathrm{EIC}} \quad$ Expected interruption cost.

$C^{\mathrm{OU}} \quad$ Cost of upgrading an existing substation to a smart substation.

$C^{\mathrm{OM}} \quad$ Maintenance and operation cost of smart substations.

\section{Variables}

$t_{s, f, l} \quad$ Interruption time of customers in substation $s$ due to a fault on line $l$ at feeder $f$, where $f \in S_{f}, s \in \Omega_{f}, l \in \Omega_{f}$.

$$
\begin{aligned}
& X_{f, s} \quad \text { Binary variable: } 1 \text { if substation } s \text { at feeder } f \text { is } \\
& \mathbf{X} \quad \text { upgraded to a smart substation; } 0 \text { if not. } \\
& \mathbf{X} \quad \text { Variable matrix with element } X_{f, s} \text {, where } \\
& f \in S_{f}, s \in \Omega_{f} \text {. }
\end{aligned}
$$

\section{INTRODUCTION}

$\mathrm{D}$ ISTRIBUTION system reliability relates to customer satisfaction, and may also lead to penalties to utilities who fail to meet the expected level of power supply reliability [1-3]. Currently, the widely adopted reliability indices, such as the system average interruption frequency index (SAIFI), system average interruption duration index (SAIDI), are primarily benchmarked based on long duration interruptions [4, 5]. Reducing the long duration interruptions is therefore a key objective in both distribution system planning and operation.

One of the most effective methods to reduce the outage time is known as smart substations [6,7]. Although there is currently a lack of consensus on the definition of smart substations, it is commonly agreed that smart substations serve as the basis and backbone of the establishment of the smart grid. The automation level deployed at a substation therefore reflects its "smartness" [8], from strengthening the station's local functions (e.g. fault isolation) to improving the integrated performance with respect to the security, economics (including loss reduction), power supply reliability, power quality and power restoration. In contrast to automatic switch, smart substations often offer certain decision-support capabilities which can be integrated into various distribution control levels (HV, MV, LV) [9], therefore also enabling various smart grid applications such as online monitoring and state estimation $[10,11]$.

Conventionally, in a distribution system with relatively limited level of automation, the sectionalizing switches (normally closed) are used to limit the impacts of a faulty condition by isolating the upstream customers from the fault. If sectionalizing switches are combined with tie switches (normally opened), the outage duration can be reduced further by re-supplying power to some interrupted downstream customers from the neighboring feeders through either automatic or manual operation. Besides, the time spent on detecting the fault location and repairing the faulty equipment can be long due to the lack of communication and other supporting functions. On the contrary, for a distribution system equipped with smart substations, the outage time can be significantly reduced, however, the effectiveness are restricted by the location and the number of smart substations [8]. It should be pointed out that there are several significant differences between a smart substation and a sectionalizing 
switch, including: a) a smart substation is regarded as an integrated solution with some smart switches, a communication infrastructure and a decision-making system, while a conventional switch is just an execution device; 2) a smart substation functions with a local area network-based system and advanced multifunctional protection and control IEDs [12], while a conventional switch is open/closed manually or remote-controlled by signals in a conventional centralized system based on RTUs and numerous protection and control devices; 3) a smart substation requires less network resources. Real-time data are collected and dealt with first in a smart substation, and then locally filtered information will be sent to a Distribution Management System (DMS) and neighboring substations. A conventional remote-controlled switch is activated by signals from DMS where all the data in the distribution system are processed in a centralized way which demands plenty of network resources.

The problem of optimally allocating smart substations is not systematically addressed in existing publications. Some papers focus on the optimal placement of remote-controlled switches so as to implement some functionalities of a smart substation. In [13], the optimal placement problem of remote-controlled switches is formulated as a weighed set-cover problem and with the objective of minimizing the total number of switches in selected basic switch groups and extended switch groups. In [14], the minimum cost of energy not supplied is achieved under reliability and flow capacity constraints, and the optimal allocation of switches is solved by the memetic algorithm. The optimal number and location of remote-controlled sectionalizing switches are optimized simultaneously in [15, 16], however the reliability index is not considered. The optimal placement of switching devices is derived to minimize the total cost of customer service outage and investment cost of line switches by employing immune algorithm (IA) in [17].

Due to the need to upgrade conventional substations to smart substations in a modern distribution system, a smart substation allocation model is developed in this study which extends the state-of-the-art research on remote-controlled switch problem. This paper mainly focuses on the application of smart substations to enhance restoration capability and reduce the outage time of customers. A smart substation has some advanced functionalities, including the automatic operation control of substations, condition based maintenance (CBM) of the devices, self-adaption of variable operation states, distributed coordinated control, smart analysis and decision-making [18]. These functionalities are implemented in a smart substation, based on the IEC61850 communication protocol. With the high-speed Ethernet, the IEC 61850-based communication system can manage all the data available at the process level as well as at the station level [19]. By using the term "smart substation", we would like to emphasize the possibility that a conventional substation can be upgraded to a substation with a specific level of "smartness" in different ways, which has to be understood from an integrated perspective, i.e. "communication + control + decision-making + operation". We regard a "smart substation" as an integrated solution driving incremental benefits in capital outlay, operation and maintenance expenses, and the benefits of customers [20]. This understanding is somewhat not highlighted enough in existing publications that focus on "optimal placement of switching devices".

A smart switching device (SSD) [21] is one of the key components in a smart substation. Different from a conventional switching device, a SSD adopts a standard communication protocol (IEC61850), and integrates condition monitoring, measurement and control, protection and communication, satisfying the demand for isolating the faulty area and restoring power supply to customers in the healthy area quickly following a fault [22]. When the optimal allocation of smart substations is carried out, the allocation of smart switches is implemented as well. The proposed method has the following features.

1) Both the number and the allocations of smart substations are optimized simultaneously. The proposed model is formulated as a mixed integer linear programming (MILP) problem which can be effectively solved by commercial solvers.

2) The System Average Interruption Duration Index (SAIDI) constraint is considered in the established model, and the recommended SAIDI value is given but can be easily adjusted according to the planned target of a utility.

3) A detailed fault clearing model is developed to minimize the EENS for the section where the faulty line exists, thus, the optimal sequence for detecting candidate faulty lines is obtained. In this work, a line failure refers to a failure associated with a conductor, with the protection and control devices attached to the conductor such as a capacitor and a fuse cutout.

4) The piecewise linear outage cost function of customers is linearized by introducing ancillary variables, and in this way the planning problem of actual large-scale distribution systems can be addressed.

The remainder of this paper is organized as follows. Section II presents the formulation of the smart substation allocation model and the linearization strategies. Section III presents numerical simulation results for two test systems. The concluding remarks are given in Section IV.

\section{MATHEMATiCAL MODEL}

In this section, a Smart Substation Allocation Model (SSAM) is first presented to minimize the cost of upgrading substations. Then, a fault clearing model is developed and the failure time estimated. Two linearization strategies applied for the failure time and interruption cost function are introduced to ensure SSAM be a mixed integer linear programming model, and could be solved effectively by commercial solvers. Finally the linearized SSAM is presented. The relationship among subsections in Section II is illustrated in Fig. 1. 


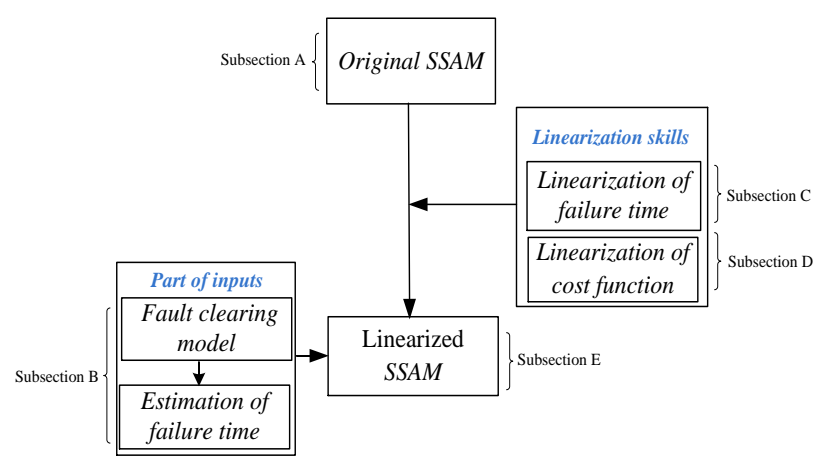

Fig. 1 The relationship among subsections in Section II

A smart substation allocation model (SSAM) is presented in subsection A. By employing the linearization skills presented in subsections C and D, the SSAM can be transformed into a mixed integer linear programming problem as detailed in subsection E. A fault clearing model is developed in subsection B so as to estimate the failure time in two cases, which serves as a part of the input of the linearized SSAM.

\section{A. Original SSAM}

Upgrading a traditional substation to a smart substation can be expensive because of the advanced devices, complex maintenance operation and some necessary communication infrastructures. It is necessary to investigate the tradeoff between the upgrade cost of substations and the customer interruption cost.

The objective of the optimal smart substation allocations is to minimize the upgrading cost of substations and the expected interruption cost of customers while keeping the index of SAIDI less than a desired threshold. The upgrading cost of substations consists of the investment $\operatorname{cost} C^{\text {OU }}$, as well as the maintenance and operation cost $C^{\mathrm{OM}}$. This problem can be formulated as,

$$
\begin{aligned}
& \text { Min } C^{\mathrm{EIC}}+C^{\mathrm{OU}}+C^{\mathrm{OM}} \\
& C^{\mathrm{EIC}}=\sum_{f \in S_{f}} \sum_{l \in \Omega_{f}} \sum_{s \in \Omega_{f}} \lambda_{f, l}\left(\sum_{k \in S_{k}} p_{f, s, k} f_{k}\left(t_{s, f, l}\right)\right) \\
& C^{\mathrm{OU}}=\sum_{f \in S_{f}} \sum_{s \in \Omega_{f}} X_{f, s}\left(C_{f, s}^{C I C}+C_{f, s}^{I C}\right) \\
& C^{\mathrm{OM}}=\sum_{f \in S_{f}} \sum_{s \in \Omega_{f}} X_{f, s} C_{f, s}^{M C}
\end{aligned}
$$

Subject to

$$
\begin{aligned}
& S_{A I D I}(\mathbf{X}) \leq S_{A I D I}^{\lim } \\
& S_{A I D I}(\mathbf{X})=\frac{\sum_{f \in S_{f}} \sum_{s \in S_{s}} \sum_{l \in S_{l}} N_{f, s} \lambda_{f, l} t_{s, f, l}}{\sum_{f \in S_{f}} \sum_{s \in S_{s}} N_{f, s}} \\
& X_{f, s} \in\{0,1\} \quad \forall f \in S_{f}, \forall s \in S_{s}
\end{aligned}
$$

Eqn (2) presents the expected interruption cost of customers. The method employed in this paper for calculating the expected interruption cost of customers is based on the method presented in [5]. Eqn (3) presents the total investment cost of smart substations for a smart substation allocation scheme which consists of investment cost and installment cost. The total investment cost is the investment cost of upgrading a conventional substation multiplied by the number of substations to be upgraded. Eqn (4) presents the total maintenance and operation cost of smart substations for a smart substation allocation scheme, including the maintenance and operation cost of upgrading a conventional substation multiplied by the number of substations to be upgraded. Eqns (2)-(4) are employed to calculate the expected interruption cost of customers, investment cost, as well as the maintenance and operation cost. Eqn (5) ensures that the SAIDI index of a distribution network be limited by the threshold. The $S_{A I D I}^{\lim }$ can be determined by the DSO, meanwhile the value should be in the range between the minimum and the maximum of SAIDI so that the problem is feasible. The range can be calculated by the method proposed in [14].

$C^{\text {EIC }}$ and $S_{A I D I}(\mathbf{X})$ are related to the outage duration which is highly dependent on the locations of smart substations that can perform automatic fault isolation and power restoration through a neighboring feeder. The optimization model described by (1)-(7) is nonlinear. To solve this model effectively, some linearization operations are implemented in subsection $\mathrm{C}$.

\section{B. Fault Clearing Model}

When a fault occurs in the distribution system, the sectionalizers will be operated to isolate the fault. The out-of-service area without fault can be reserved in a certain time depending on operation time of switches, while the out-of-service area with fault can obtain power only when the fault is cleared. The outage time of the faulty area is related to how the maintenance process works.

In the actual maintenance process, the first thing to do for the DSO operators is to identify the location of fault, which is not easy without the communication between the distribution system center and the substations in a faulty area. In this case, the staff members will check the suspected lines until the line with fault is located. The operation of detecting usually begins from the upstream of the fault area to the downstream in sequence, which can be time-consuming. The out-of-service time of the fault area is therefore the sum of the time spent on detecting the candidate lines and repairing the faulty line and resupplying power to the outage areas. The proper sequence of detecting candidate lines can effectively locate the faulty lines, thus it can be beneficial to reducing the out-of-serve time.

The section concept is introduced in [14], and the candidate faulty lines are confined in a certain section. Thus, in this part, an optimal fault clearing model is developed to minimize the EENS for any section $z$, and described as,

$$
\text { Min } E_{z}^{E N S}=\mathbf{\Upsilon}_{z}^{\prime}\left(\left(\mathbf{D}_{z} \cdot \mathbf{G}_{z} \cdot \mathbf{D}_{z}{ }^{\prime}\right)^{\prime} \mathbf{T}_{z}^{d t}+\mathbf{T}_{z}^{r t}+\mathbf{T}_{z}^{a t}\right) \cdot p_{z}
$$

where $\left(\mathbf{D}_{z} \cdot \mathbf{G}_{z} \cdot \mathbf{D}_{z}{ }^{\prime}\right)^{\prime} \mathbf{T}_{z}^{d t}$ is a time vector of finding the faulty line in section $z$ according to the detect sequence matrix $\mathbf{D}_{z}$. The introduction of $\mathbf{G}_{z}$ is to obtain a new matrix $\mathbf{D}_{z} \cdot \mathbf{G}_{z} \cdot \mathbf{D}_{z}{ }^{\prime}$ which acts as an indicator of whether a candidate line is detected before the faulty line is located in section $z$.

In this formulation, some assumptions have been made as: 
1) Any line can only be detected once, so constraint (9) must be respected.

$$
\sum_{t}^{T_{z}} D_{z, l, t}=1 \quad, l \in \Omega_{f, z}
$$

2) Only one line can be detected at each time interval, so constraint (10) must be respected.

$$
\sum_{l=1}^{n_{z}} D_{z, l, t}=1 \quad, t=1,2, \ldots T_{z}
$$

By solving (8)-(10), the optimal detecting sequence of candidate lines can be obtained and the corresponding minimum EENS attained. Thus, the expected failure durations of customers in section $\mathrm{z}$ can be estimated.

To sum up, the failure duration can be described as

$$
t_{s, f, l}^{\text {cur }}=\left\{\begin{array}{l}
0, \quad \forall s \notin \Omega_{f} \\
t_{z}^{\text {uss }}, \forall l \in \Omega_{f, z}, \forall s \in \Omega_{f, z}^{\text {up }} \\
t_{z}^{\text {uss }}+t_{z}^{\mathrm{dts}}, \forall l \in \Omega_{f, z}, \forall s \in \Omega_{f, z}^{\text {down }} \\
t_{z}^{\text {uss }}+t_{z}^{\mathrm{dts}}+\overline{\mathbf{\Upsilon}}_{z, l}^{\prime}\left(\left(\mathbf{D}_{z} \cdot \mathbf{G}_{z} \cdot \mathbf{D}_{z}\right)^{\prime} \mathbf{T}_{z}^{d t}+\mathbf{T}_{z}^{r t}+\mathbf{T}_{z}^{a t}\right), \forall s, l \in \Omega_{f, z}
\end{array}\right.
$$

If substation $s$ is not supplied by feeder $f$, the failure on line $l$ in feeder $f$ will have no impacts on the power supply of substation $s$.

If substation $s$ is supplied by feeder $f$ and located in the upstream of section $z$ supplied by feeder $f$ where the faulty line $l$ lies in, the outage time is $t_{z}^{\text {uss }}$.

If substation $s$ is supplied by feeder $f$ and located in the downstream of section $z$ supplied by feeder $f$ where the faulty line $l$ lies in, the outage time is $t_{z}^{\text {uss }}+t_{z}^{\mathrm{dts}}$.

If substation $s$ is supplied by feeder $f$ and located within the same section $z$ with the faulty line $l$, then the outage time of the customers is $t_{z}^{\text {uss }}+t_{z}^{\mathrm{dts}}+\overline{\mathbf{\Upsilon}}_{z, l}^{\prime}\left(\left(\mathbf{D}_{z} \cdot \mathbf{G}_{z} \cdot \mathbf{D}_{z}\right)^{\prime} \mathbf{T}_{z}^{d t}+\mathbf{T}_{z}^{r t}+\mathbf{T}_{z}^{a t}\right)$.

In another case, if the substations in the distribution system are smart ones, each line and substation will be an independent section, thus the outage duration can be attained by

$$
t_{s, f, l}^{\text {smart }}=\left\{\begin{array}{l}
0, \quad \forall s \notin \Omega_{f} \\
t_{z}^{\text {uss_a }}, \forall l \in \Omega_{f, z}, \forall s \in \Omega_{f, z}^{\text {up }} \\
t_{z}^{\text {uss_a }}+t_{z}^{\text {dts_a }}, \forall l \in \Omega_{f, z}, \forall s \in \Omega_{f, z}^{\text {down }} \\
t_{z}^{\text {uss_a }}+t_{z}^{\text {dts_a }}+\left(t_{z, l}^{d t}+t_{z, l}^{r t}+t_{z, l}^{a t}\right), \forall s, l \in \Omega_{f, z}
\end{array}\right.
$$

When a fault occurs, a smart substation can locate the fault by analyzing the electrical information collected by the monitoring devices. Control signals will be sent to the smart switches, so that the faulty area can be identified and isolated by opening the normally closed smart switches. With the help of smart substations, the faulty area can be located accurately and quickly. It takes less time for a maintenance team to repair all the defective network components. Eqns (11) and (12) embody this process and are employed to estimate the failure duration in a distribution system with conventional substations and smart substations, respectively.

\section{Linearization of Failure Time}

The failure duration is effected by the location of the smart substations. The relation between failure duration and location and types of substations is introduced in the above content as in subsection B. By introducing the following two constraints, these relations can be modeled in a linear form [15] and added into the SSAM.

$$
\begin{aligned}
& t_{s, f, l} \geq t_{s, f, l}^{\mathrm{smart}} \\
& t_{s, f, l} \geq t_{s, f, l}^{\mathrm{cur}}\left[1-\sum_{i \in \Omega_{f, l, s}^{s s}} X_{f, i}\right]
\end{aligned}
$$

A part of the objective of the SSAM is to minimize the expected interruption cost of customers which is a piecewise-linear function of the interruption time. The interruption time is equal to the minimum of $t_{s, f, l}^{\text {smart }}$ and $t_{s, f, l}^{\mathrm{cur}}\left[1-\sum_{i \in \Omega_{f, l, s}^{s s}} X_{f, i}\right]$ as in (13) and (14). If there is at least one smart substation between the faulty line $l$ and the customers in substation $s$, the interruption time is equal to $t_{s, f, l}^{\text {smart }}$, otherwise the interruption time is equal to $t_{s, f, l}^{\mathrm{cur}}$.

As has been pointed out previously, the out-of-service area without fault in the downstream can be isolated from the fault by opening the sectionalizing switch and resupplied by closing the combined tie switch. Therefore, if there exists more than one smart substation located between substations on each side of a tie switch and its feeders which supply power to these substations, at least one of the substations that lie on each side of the tie switch is smart. If none of the substations between substations on each side of a tie switch and its feeders that supply power to these substations is smart, substations that lie on each side of the tie switch are not smart ones. These relations can be described as follows.

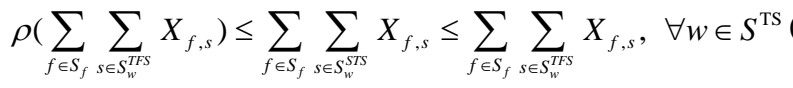

\section{Linearization of Interruption Cost Function}

The cost function of outage is represented as a piecewise function. In [17], the service interruption cost and key customers are introduced. The interruption cost function of customers with outage duration $t_{s, f, l}\left(\forall f \in S_{f}, \forall s, l \in \Omega_{f}\right)$ can generally be described as $f\left(t_{s, f, l}\right)=a_{g} t_{s, f, l}+b_{g}, t_{g} \leq \forall t_{s, f, l}<t_{g+1}, \forall g \in\left\{1, \ldots, N_{g}-1\right\}$ (16)

The main work in this part is on transforming the piecewise function into continual, differential function by introducing mixed integer ancillary variables.

The interruption cost function of customers with type $k$ and interruption time $t_{s, f, l}$ is depicted in Fig. 2 and described as

$$
\begin{gathered}
f_{k}\left(t_{s, f, l}\right)=\sum_{g=1}^{N_{g}} \gamma_{s, k, f, l, g} y_{k, g} \\
t_{s, f, l}=\sum_{g=1}^{N_{g}} \gamma_{s, k, f, l, g} a_{k, g}^{\text {add }} \\
\gamma_{s, k, f, l, g} \leq z_{s, k, f, l, g}, \quad \forall g \in\left\{1,2, \ldots, N_{G}\right\} \\
\gamma_{s, k, f, l, g} \geq 0, \quad \forall g \in\left\{1,2, \ldots, N_{G}\right\} \\
z_{s, k, f, l, g} \in\{0,1\}, \forall g \in\left\{1,2, \ldots, N_{G}\right\}
\end{gathered}
$$




$$
\begin{gathered}
\sum_{g=1}^{N_{g}} z_{s, k, f, l, g} \leq 2 \\
z_{s, k, f, l, g_{1}}+z_{s, k, f, l, g_{2}} \leq 1, \quad 1 \leq \forall g_{1}<N_{G}-1, g_{1}+1<\forall g_{2} \leq N_{G}
\end{gathered}
$$

It is shown in Fig. 2 that if the interruption time $t_{s, f, l}$ of customers with type $k$ is in $\left[t_{k, g}^{\text {add }}, t_{k, g+1}^{\text {add }}\right]$, both of the indicative variables $z_{s, k, f, l, g}$ and $z_{s, k, f, l, g+1}$ are 1 . The interruption time $t_{s, f, l}$ can be rewritten in a linear formulation of $t_{k, g}^{\text {add }}$ and $t_{k, g+1}^{\text {add }}$ with coefficients $\gamma_{s, k, f, l, g}$ and $\gamma_{s, k, f, l, g+1}$. Besides, the interruption cost $f_{k}\left(t_{s, f, l}\right)$ can also be written in a linear formulation of $y_{k, g}$ and $y_{k, g+1}$ with coefficients $\gamma_{s, k, f, l, g}$ and $\gamma_{s, k, f, l, g+1} \cdot$

By substituting (17) into (2), the interruption cost of customers can be described as

$$
C^{\mathrm{EIC}}=\sum_{f \in S_{f}} \sum_{l \in \Omega_{f}} \sum_{s \in \Omega_{f}}\left[\lambda_{f, l} \sum_{k \in S_{k}} p_{f, s, k}\left(\sum_{g=1}^{N_{g}} \gamma_{s, k, f, l, g} y_{k, g}\right)\right]
$$

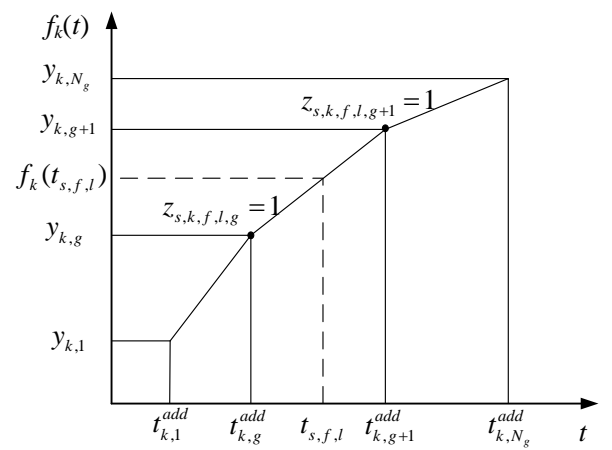

Fig. 2 Piecewise linear function of the interruption cost for customers with type $k$

\section{E. Linearized SSAM}

To sum up, the SSAM can be described as a mixed integer linear programming problem and presented as follows.

\section{Objective function (1)}

Subject to (5)-(7), (13)-(15), (18)-(23)

The terms in (1) can be attained by (3), (4), and (22).

The proposed linearized SSAM in this paper is applicable for balanced distribution systems, and can be expanded for distribution systems with unbalanced feeders. In the model for three-phase unbalanced distribution systems, some parameters and variables are attached with phase sequence indices, while in the one for balanced distribution systems there are not phase sequence indices associated. A modified smart substation allocation model is added for three-phase unbalanced distribution systems, as detailed below:

Objective function (1)

Subject to (5), (7), (15)

$$
S_{A I D I}(\mathbf{X})=\frac{\sum_{\varphi \in S_{\varphi}} \sum_{f \in S_{f}} \sum_{s \in S_{s}} \sum_{l \in S_{l}} N_{f, s, \varphi} \lambda_{f, l, \varphi} t_{s, f, l, \varphi}}{\sum_{\varphi \in S_{\varphi}} \sum_{f \in S_{f}} \sum_{s \in S_{s}} N_{f, s, \varphi}}
$$

$$
\begin{array}{r}
t_{s, f, l, \varphi} \geq t_{s, f, l, \varphi}^{\mathrm{smart}} \\
t_{s, f, l, \varphi} \geq t_{s, f, l, \varphi}^{\mathrm{cur}}\left[1-\sum_{i \in \Omega_{f, l, s}^{s s}} X_{f, i}\right] \\
t_{s, f, l, \varphi}=\sum_{g=1}^{N_{g}} \gamma_{s, k, f, l, \varphi, g} t_{k, g}^{\text {add }} \\
\gamma_{s, k, f, l, \varphi, g} \leq z_{s, k, f, l, \varphi, g}, \quad \forall g \in\left\{1,2, \ldots, N_{G}\right\} \\
\gamma_{s, k, f, l, \varphi, g} \geq 0, \quad \forall g \in\left\{1,2, \ldots, N_{G}\right\} \\
z_{s, k, f, l, \varphi, g} \in\{0,1\}, \forall g \in\left\{1,2, \ldots, N_{G}\right\} \\
\sum_{g=1}^{N_{g}} z_{s, k, f, l, \varphi, g} \leq 2 \\
z_{s, k, f, l, \varphi, g_{1}}+z_{s, k, f, l, \varphi, g_{2}} \leq 1, \quad 1 \leq \forall g_{1}<N_{G}-1, \\
g_{1}+1<\forall g_{2} \leq N_{G}
\end{array}
$$

where $\varphi$ and $S_{\varphi}$ are respectively the index and set of phases. All symbols in Eqns (24)-(32) with index $\varphi$ have the same meanings as their corresponding symbols without index $\varphi$ introduced in the nomenclature section.

In this paper, the commercial solver CPLEX [23] is employed to obtain the global solution because the allocation problem of smart substations is formulated as a MILP model.

\section{CASE Study}

In this section, the proposed method is applied to two power systems including the IEEE RBTS-Bus 4 testing network [4] and a practical $10 \mathrm{kV}$ medium voltage network of Denmark.

The investment cost of upgrading an existing substation to a smart one is approximately $\$ 22677$ with a life cycle of 15 years. Suppose the annual maintenance and operation cost is $2 \%$ of the annualized investment cost [16], the total annual cost for upgrading one substation is $\$ 1542.04$.

In this work, a line failure refers to a failure associated with a conductor, as well as with protection and control devices attached to the conductor such as a capacitor and a fuse cutout. In each fault scenario, one line failure is considered with the failure rate set as the sum of the failure rate of a conductor and that of the protection and control devices attached to the conductor, which are respectively specified as 0.01 failures/year.km and 0.025 failures/year. The service interruption cost of customers with different types is obtained from $[4,17] . \rho$ is set as 0.00001 . The load demand employed in the study cases is the average value in each of the 15 years with an annual load growth rate 5\% [24].

The time related to the repair model is shown in Table I.

TABLE I

Time Parameters Related to FAUlt Clearing Model

\begin{tabular}{c|c|c}
\hline Parameters & Value & Unit \\
\hline Detect time of each line & 1 & Hour \\
\hline Repair time of each line & 3 & Hours \\
\hline $\begin{array}{c}\text { Restoration time of a faulty section } \\
\text { after the faulty line is repaired }\end{array}$ & 0.5 & Hour \\
\hline Upstream switch time (manual) & 0.9 & Hour \\
\hline
\end{tabular}




\begin{tabular}{c|c|c}
\hline Downstream switch time (manual) & 0.45 & Hour \\
\hline Upstream switch time (automatic) & 0.2 & Hour \\
\hline Downstream switch time (automatic) & 0.2 & Hour \\
\hline
\end{tabular}

Simulation tests are implemented in an Intel i5 $3.4-\mathrm{GHz}$ personal computer with 8 GB RAM and Windows 7 Enterprise as the operating system. The algorithms are implemented in AMPL, which is an algebraic modeling language to describe mathematical problems [23]. AMPL/CPLEX, a highly efficient commercial solver, is employed to solve the developed model [25].

\section{A. RBTS-Bus 4}

RBTS-BUS 4 is a standard test system and is employed to demonstrate the proposed method in this paper. The average load, number and type of customers on each feeder, length of each line and topology network are given in [4]. The threshold of SAIDI is set as 20 (min/year).

The running time of the proposed solution strategy is 0.13 second. The optimal number of smart substations to be upgraded is 8 which are labeled by red numbers shown in Fig. 3. Each dashed line in Fig. 3 represents a tie switch. Substations with three kinds of colors, i.e. blue, yellow, green, supply three types of customers, i.e. residential, small and commercial customers, respectively. In addition, a detailed dependency analysis between the number of smart substations and the resulted performance is shown in Fig. 4. It can be easily observed both outage cost and SAIDI decreases as the number of smart substation increases. However, when the investment is either oversized or undersized, the economic viability will be easily lost. As an example, for a scenario with $100 \%$ smart substations (i.e. 38 smart substations), the total cost is $\$$ 65308.05 , which is 1.95 times of the total cost for the optimal solution (\$33477.2), although it can reduce the SAIDI from 12.15 to 2.39 . Comparing to the scenario with no smart substation at all, the optimal solution can achieve a saving by $14.2 \%$ and reduce the SAIDI from 15.76 to 12.15 .

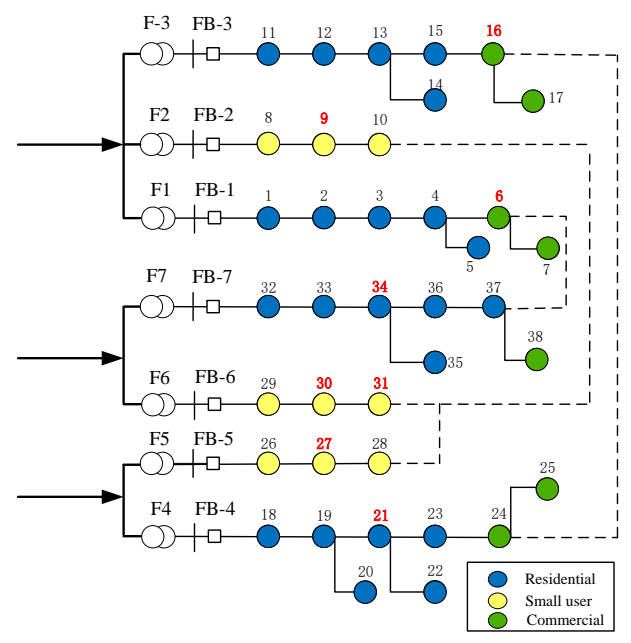

Fig. 3 Optimal number and allocations of smart substations for RBTS-BUS 4

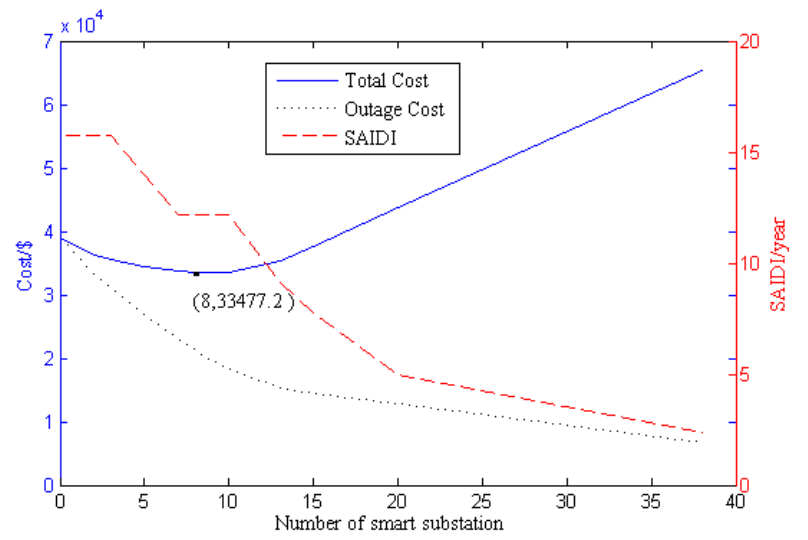

Fig. 4 The total cost, outage cost and SAIDI with different number of smart substations in the RBTS-Bus 4 distribution system

\section{B. Medium Voltage Distribution Network in Denmark}

To demonstrate the applicability of the proposed approach for actual power systems, a medium voltage distribution network in the east of Denmark is served for demonstration, as depicted in Fig. 5. There are 5 primary substations and 98 $10 / 0.4 \mathrm{kV}$ secondary substations. The power grid serves approximately 5000 customers. There are 26 categories of customer types in the Danish distribution grid, which in this paper are regrouped into 3 types: Residential, Commercial Industrial. The threshold of SAIDI is set as 50 (min/year). The optimal allocations of smart substations with its numbers marked red are shown in Fig. 5.

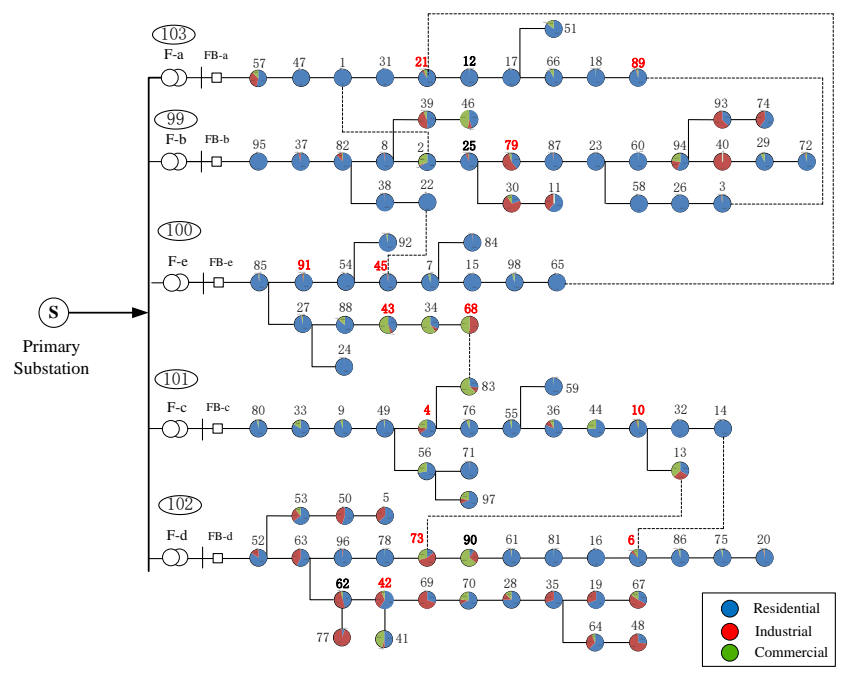

Fig. 5 Optimal number and allocations of smart substations in an actual medium voltage distribution network in Denmark

The running time of the proposed solution strategy is 3.21 second. The minimum total cost is $\$ 49749.23$ and the optimal number of smart substations is 12 which is shown in Fig. 5. Similar to results achieved in case A, it is observed that the upgrading of substations can effectively decrease the outage cost. If the number of smart substations is not more than 12 , the total cost decreases as the number of smart substations increases. 


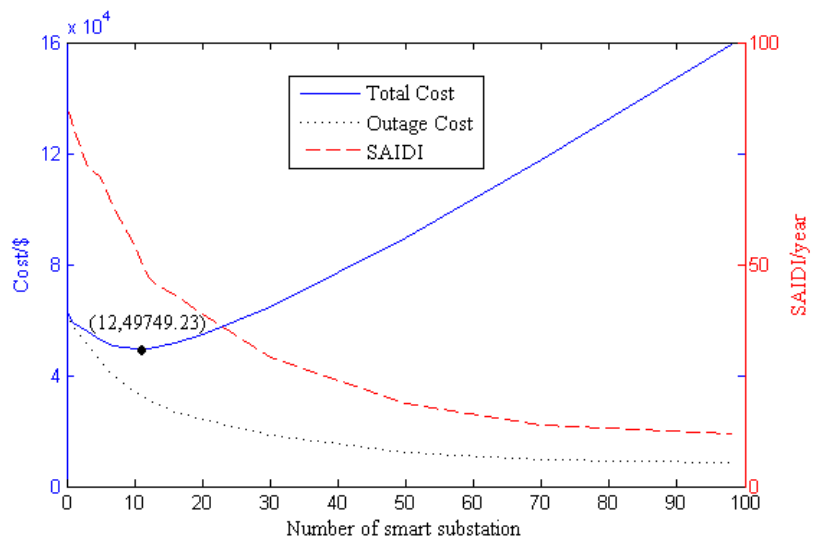

Fig. 6 Total cost, outage cost and SAIDI with different number of smart substations in an actual medium voltage distribution network in Denmark

\section{Sensitivity analysis}

The change of line failure rates, investment cost of upgrading the substation, threshold value of SAIDI and the method of estimating the interruption cost of customers have great impacts on the optimal solution achieved by SSAM. A sensitivity analysis on some selected parameters is investigated for Case B grid in this section. Due to space limitation of the paper, the attention is focused on the impacts of the failure rates and cost of upgrading a substation to a smart substation in the proposed model.

Since all the distribution lines are cables in the network, they have lower failure rates than overhead lines [4]. Table II gives results associated with some cases with different changes in failure rates. From the results, it can be seen that number of substation to be upgraded increases as the failure rates increase. For example, Number of substations to be upgraded is almost doubled when failure rates increase by $50 \%$.

TABLE II

IMPACTS OF FAILURE RATES ON SOLUTIONS OF THE SSAM

\begin{tabular}{c|c|c|c|c}
\hline $\begin{array}{c}\text { Failure rates } \\
\text { changes }\end{array}$ & $\begin{array}{c}\text { Number } \\
\text { of SS }\end{array}$ & Outage cost $(\$)$ & Total cost $(\$)$ & SAIDI \\
\hline$-50 \%$ & 3 & 25602.5 & 30228.64 & 35.96 \\
\hline$-25 \%$ & 8 & 28225.6 & 40561.90 & 43.57 \\
\hline--- & 12 & 31244.8 & 49749.23 & 47.25 \\
\hline$+25 \%$ & 17 & 32895 & 59109.72 & 50 \\
\hline$+50 \%$ & 23 & 33789.7 & 69256.65 & 49.81 \\
\hline
\end{tabular}

The investment cost of upgrading a conventional substation to a smart substation also has impacts on the optimal solution of SSAM. It is always decreasing because of the advances in knowledge, technology and mass production of concerned devices. Different upgrading costs are applied to the developed model and the results are shown in Table III. As can be seen, more substations can be upgraded with less outage cost and lower SAIDI as the upgrading cost decreases.

TABLE III

\begin{tabular}{c|c|c|c|c}
\multicolumn{5}{c}{ IMPACTS OF SS COSTS ON SOLUTIONS OF THE SSAM } \\
\hline $\begin{array}{c}\text { SS cost } \\
\text { changes }\end{array}$ & $\begin{array}{c}\text { Number } \\
\text { of SS }\end{array}$ & $\begin{array}{c}\text { Outage } \\
\text { cost }\end{array}$ & $\begin{array}{c}\text { Total } \\
\text { cost }\end{array}$ & SAIDI \\
\hline$-50 \%$ & 17 & 26086.7 & 39194.04 & 41.62 \\
\hline$-25 \%$ & 13 & 29922.4 & 44957.30 & 45.54 \\
\hline
\end{tabular}

\begin{tabular}{c|c|c|c|c}
\hline--- & 12 & 31244.8 & 49749.23 & 47.25 \\
\hline$+25 \%$ & 11 & 32782 & 53985.07 & 49.93 \\
\hline$+50 \%$ & 11 & 32782 & 58225.68 & 49.93 \\
\hline
\end{tabular}

\section{Comparison of two strategies}

Two different strategies, i.e. the optimal allocations of smart substations (SS) and remote-controlled switches (RCS), are compared. The cost setting of a remote-controlled switch is $\$$ 9071 [17]. The upstream and downstream remote-controlled switch time settings are $0.45 \mathrm{~h}$ and $0.35 \mathrm{~h}$ respectively in the remote-controlled switch allocation model with the consumed network resources considered. Comparison results are shown in Table IV.

TABLE IV

COMPARISONS BETWEEN THE OPTIMAL ALLOCATIONS OF SS AND RCS

\begin{tabular}{c|c|c|c|c}
\hline Strategies & $\begin{array}{c}\text { Number of } \\
\text { SS/RCS }\end{array}$ & $\begin{array}{c}\text { Outage } \\
\text { cost }(\$)\end{array}$ & $\begin{array}{c}\text { Total } \\
\text { cost (\$) }\end{array}$ & SAIDI \\
\hline Allocation of SS & 12 & 31244.8 & 49749.23 & 47.25 \\
\hline $\begin{array}{c}\text { Allocation of } \\
\text { RCS }\end{array}$ & 19 & 31935.3 & 43655.00 & 49.38 \\
\hline
\end{tabular}

From Table IV it is clear that the optimal number of SS is much smaller than that of RCS; however, the optimal smart substation allocation strategy leads to less outage cost than the remote-controlled switch allocation strategy due to the decrease of the outage duration. Because of the high cost of upgrading an existing conventional substation to a smart one, the total cost of the smart substation allocation strategy is higher than that of the remote-controlled switch allocation strategy. It is expected that the cost of a smart substation will be decreased with the continuous development of technology, and hence more wide applications of smart substations are anticipated.

\section{E. Discussions}

Many factors, such as load demand increases, the penetration of DERs, implementations of demand response programs, new feeders and/or transformers, and various types of customers, have impacts on the optimal allocations of smart substations. The load demand employed in the study cases is the average value in each of the 15 years with an annual load growth rate $5 \%$.

The penetration of DERs can contribute to reducing the outage duration or even avoiding the outage of customers by providing power supply following a fault. Incentives for consumers to participate in a demand response program may be provided as well [26]. Adding a new feeder or transformer to an existing distribution system is beneficial to reducing the outage duration. All these three factors can affect the outage duration, which is an important parameter in the proposed smart substation allocation model.

For given locations and sizes of DERs, the demand response program and adding-new-feeder planning, the outage duration of customers in a distribution system can be estimated by the proposed method in subsection $\mathrm{C}$ of Section II. The three factors, including the penetration of DERs, impacting of demand response programs, adding new feeders and/or 
transformers, have impacts on the outage duration, and further the optimal allocation solution of smart substations. The presented optimization model for allocating smart substations has some degree of flexibility and can accommodate some practical factors specific to various utilities.

In the given study cases, customers are grouped into 3 types. Different types of customers have different service interruption costs. The priority degrees of various customers have impacts on the allocations of smart substations with regard to various outage costs of customers. The impact is clearly shown in Fig.5. Generally, customers with larger service interruption costs have stronger demands for smart substations. Substations 34, 43, 68 in feeder F-e supply power for most of the commercial customers, and will not be cut off even if there is a faulty component in other areas supplied by feeder F-e; the fault can be isolated by the smart substation 43 , and substations 34,43 , 68 can be re-supplied quickly by feeder F-c. In the same way, with the help of smart substations 79 and 89 , the interruption time of substations 40, 74, 79, 93 which supply power for most of the industrial customers is less than that in the case if substations 79 and 89 are both conventional ones.

\section{CONCLUDING REMARKS}

A smart substation allocation model is developed in this paper to investigate the impacts of employing smart substations on reducing the outage time of interrupted customers. The objective of the SSAM is set as minimizing the upgrade cost of substations and the expected interruption cost of customers while maintaining the SAIDI index less than its threshold. Numerical results demonstrate that upgrading conventional substations to smart substations can reduce the outage time and expected interruption cost of customers. The impacts of line failure rates and smart substation costs on solutions of the SSAM are also examined, which helps the DSO to predict other future possible scenarios. This work provides a flexible yet robust smart substation allocation method for distribution systems with different parameters.

The smart substation concept has becoming more and more widely accepted. It is of great importance to extend the proposed allocation approach while taking the other applications of smart substations into account such as minimizing power loss and voltage deviation so as to demonstrate the value of smart substations for improving the distribution network performance on a daily basis. This represents one of our future research efforts.

\section{APPENDIX A}

To elaborate the linearization method of the failure time presented in Eqns (13) and (14), a simplified distribution system topology shown in Fig. 7 is employed, with 5 lines and 5 substations. A fault occurred on line $l_{2-3}$ between substations 2 and 3 is employed for illustration.

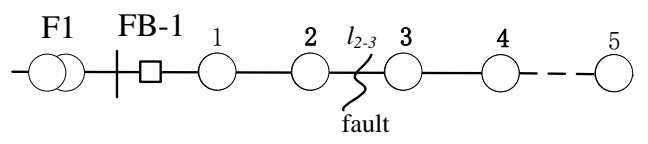

Fig. 7 A simplified distribution topology consisting of 5 lines and 5 substations.

Take the interruption time of substation 1 as an example to elaborate the linearization method of the failure time presented in Section II. Eqns (13) and (14) in the Section II can be rewritten as

$$
\begin{gathered}
t_{1, \mathrm{~F}_{1}, l_{2-3}} \geq t_{1, \mathrm{~F}_{1}, l_{2-3}}^{\mathrm{smart}} \\
t_{1, \mathrm{~F}_{1}, l_{2-3}} \geq t_{1, \mathrm{~F}_{1}, l_{2-3}}^{\mathrm{cur}}\left[1-\sum_{i \in S_{\mathrm{F}, 1, l_{-3}, 1}} X_{f, i}\right]
\end{gathered}
$$

If at least one of the two substations 1 and 2 is a smart one, Eqn (A2) can be relaxed because the value of $t_{1, \mathrm{~F}_{1}, l_{2-3}}^{\text {sart }}$ is larger than that of $t_{1, \mathrm{~F}_{\mathrm{F}}, L_{2-3}}^{\mathrm{cur}}\left[1-\sum_{i \in \Omega_{\mathrm{F}, l_{2-3}, 1}^{S S}} X_{f, i}\right]$. Then the constraint of the failure time represented by Eqns (A1) and (A2) can be rewritten as Eqn (A3).

$$
t_{1, \mathrm{~F}_{1}, l_{2-3}} \geq t_{1, \mathrm{~F}_{1}, l_{2-3}}^{\mathrm{smart}}
$$

If both substations 1 and 2 are not smart ones, Eqn (A2) can be rewritten as Eqn (A4).

$$
t_{1, \mathrm{~F}_{1}, l_{2-3}} \geq t_{1, \mathrm{~F}_{1}, l_{2-3}}^{\mathrm{cur}}
$$

Because $t_{1, \mathrm{~F}_{\mathrm{F}}, l_{2-3}}^{\text {ssart }}$ is smaller than $t_{1, \mathrm{~F}_{1}, l_{2-3}}^{\text {cur }}$, Eqn (A1) is relaxed. The constraint of the failure time represented by Eqns (A1) and (A2) can be rewritten as Eqn (A4).

\section{REFERENCES}

[1] N. Z. Xu and C. Y. Chung, "Reliability evaluation of distribution systems including vehicle-to-home and vehicle-to-grid," IEEE Trans. Power Syst., vol. 31, no.1, pp. 759-768, Jan. 2016.

[2] B. Falahati and Y. Fu, "Reliability assessment of smart grids considering indirect cyber-power interdependencies," IEEE Trans. Smart Grid, vol. 5, no. 4, pp. 1677-1685, Jul. 2014.

[3] S. Junlakarn and M. Ilić, "Distribution system reliability options and utility liability," IEEE Trans. Smart Grid, vol. 5, no. 5, pp. 2227-2234. Sep. 2014.

[4] R. N. Allan, R. Billinton, I. Sjarief, L. Goel, and K. S. So, "A reliability test system for educational purposes-basic distribution system data and results," IEEE Trans. Power Syst., vol. 6, no. 2, pp. 813-820, May 1991.

[5] R. Billinton and P. Wang, "Distribution system reliability cost/worth analysis using analytical and sequential simulation techniques," IEEE Trans. Power Syst., vol. 13, no. 4, pp. 1245-1250, Nov. 1998.

[6] V. C. Güngör, D. Sahin, T. Kocak, S. Ergüt, C. Buccella, C. Cecati, and G. P. Hancke, "Smart grid technologies: communication technologies and standards," IEEE Trans. Ind. Informat., vol. 7, no. 4, pp. 529-539, Nov. 2011.

[7] H. Farhangi, "The path of the smart grid," IEEE Power Energy Mag., vol. 8, no. 1, pp. 18-28, Jan./Feb. 2010. 
[8] L. Jakobsen, "Automation in cable distribution network $(10 \mathrm{kV})$," in Proc. the $21^{\text {st }}$ Int. Conf. and Exhibition on Electricity Distrib., Frankfurt, Germany, 2011, pp. 1-4.

[9] P. P. Parikh, M. G. Kanabar, and T.S. Sidhu, "Opportunities and challenges of wireless communication technologies for smart grid applications," in Proc. IEEE Power Energy Soc. Gen. Meeting, Minneapolis, MN, USA, 2010, pp. 1-7.

[10] C. Gomez-Quiles, A. Gomez-Expósito, and A. de la Villa Jaen, "State Estimation for smart distribution substations," IEEE Trans. Smart Grid, vol. 3, no. 2, pp. 986-995, Jun. 2012.

[11] Y. Yang, L.L. Song, H.O. Cao, Y.B. Guan, Y. Wu, X. Gao, L. Gao, and Y.B. Yuan, "Smart substation secondary system visualization and intelligent diagnosis based on improved SCD model," Int. Conf. on Renewable Power Gener. (RPG 2015), Beijing, China, 2015, pp. 1-5.

[12] C. Gomez-Quiles, A. Gomez-Expósito, and A. de la Villa Jaen, "State Estimation for smart distribution substations," IEEE Trans. Smart Grid, vol. 3, no. 2, pp. 986-995, Jun. 2012.

[13] Y. Xu, C. C. Liu, K. P. Schneider, and D. T. Ton, "Placement of remote-controlled switches to enhance distribution system restoration capability," IEEE Trans. Power Syst., vol. 31, no. 2, pp. 1139-1150, Mar. 2016.

[14] L. S. de Assis, J. F. V. González, F. L. Usberti, C. Lyra, C. Cavellucci, and F. J. Von Zuben, "Switch allocation problems in power distribution systems," IEEE Trans. Power Systs., vol. 30, no. 1, pp. 246-253, Jan. 2015.

[15] O. K. Siirto, A. Safdarian, M. Lehtonen, and M. Fotuhi-Firuzabad, "Optimal distribution network automation considering earth fault events," IEEE Trans. Smart Grid, vol. 6, no. 2, pp. 1010-1018, Mar. 2015.

[16] A. Abiri-Jahromi, M. Fotuhi-Firuzabad, M. Parvania, and M. Mosleh, "Optimized sectionalizing switch placement strategy in distribution systems," IEEE Trans. Power Del., vol. 27, no. 1, pp.362-370, Jan. 2012.

[17] C.-S. Chen, C.-H. Lin, H.-J. Chuang, C.-S. Li, M.-Y. Huang, and C.-W. Huang, "Optimal placement of line switches for distribution automation systems using immune algorithm." IEEE Trans. Power Syst., vol. 21, no. 3, pp. 1209-1217, Aug. 2006.

[18] Q. Huang, S. Jing, J. Li, D.S. Cai, J. Wu and W. Zhen, "Smart substation: state of art and future development," IEEE Trans. Power Del., to be published.

[19] D.M.E. Ingram, P. Schaub, R.R. Taylor, D.A. Campbell. Performance Analysis of IEC 61850 Sampled Value Process Bus Networks. IEEE Transactions on Industrial Informatics, Vol. 9 (3), 2013, pp. 1445-1454.

[20] J. Fan and S. Borlase, "The evolution of distribution," IEEE Power and Energy Magazine, vol. 7, no. 2, pp. 63-68, Mar. 2009.

[21] Q/GDW Z 410-2010. Technical Guide for Smart Electric Equipment. State Grid Cooperation of China (SGCC), 2010 (in Chinese).

[22] H. W. Li, L. X. Wang. Research on Technologies in Smart Substation. International Conference on Smart Grid and Clean Energy Technologies (ICSGCE), Chengdu, China, 2011, pp. 113 - 119.

[23] R. Fourer, D. M. Gay, and B. W. Kernighan, "A modeling language for mathematical programming," Manage. Sci., vol. 36, no. 5, pp. 519-554, May 1990.

[24] S. Dehghan, N. Amjady, and A. Kazemi, "Two-stage robust generation expansion planning: A mixed integer linear programming model," IEEE Trans. Power Syst., vol. 29, no. 2, pp. 584-597, Mar. 2014.

[25] IBM Corp., IBM ILOG AMPL Version 12.2 User's Guide, 2010.

[26] J. Domínguez, J. P. Chaves-Ávila, T. G. S. Román and C. Mateo, "The economic impact of demand response on distribution network planning," 2016 Power Systems Computation Conference (PSCC), Madrid, Spain, 2016, pp. 1-7.

Lei Sun received his B.E. degree in Electrical Engineering from East China University of Science and Technology, Shanghai, China, in 2012. He is currently pursuing the Ph.D. degree in the College of Electrical Engineering, Zhejiang University, Hangzhou, China.

His main research interests include power system restoration and distribution automation.
Shi You received his M.Sc. and Ph.D. in electrical engineering from Chalmers Institute of Technology, Sweden in 2007 and Technical University of Denmark in 2011 respectively. Currently, he is a research scientist at the Center for Electric Power and Energy, Department of Electrical Engineering, Technical University of Denmark.

His main research interests include market-based control, management and integration of distributed energy resources, planning, operation and management of active distribution networks and integrated energy systems.

Junjie Hu received the M.Sc degree in control theory and control engineering from Tongii University, China in 2010 and the PhD degree in Electrical Engineering from Technical University of Denmark, Denmark in 2014. Currently, he is a postdoctoral researcher with the Department of Electrical Engineering, Technical University of Denmark.

His main research interests include integration of distributed energy resources (DERs), active distribution grid operation and management, and DER's participations in ancillary service markets.

Fushuan Wen received the B.E. and M.E. degrees from Tianjin University, Tianjin, China, in 1985 and 1988, respectively, and the Ph.D. degree from Zhejiang University, Hangzhou, China, in 1991, all in electrical engineering.

He joined the faculty of Zhejiang University in 1991, and has been a full professor and the director of the Institute of Power Economics and Information since 1997, and the director of Zhejiang University-Insigma Joint Research Center for Smart Grids since 2010. He had been a university distinguished professor, the deputy dean of the School of Electrical Engineering and the director of the Institute of Power Economics and Electricity Markets in South China University of Technology, Guangzhou, China, from 2005 to 2009. Since May 2014, he has been a professor with Universiti Teknologi Brunei (Brunei University of Technology), Brunei, on leave from Zhejiang University. His research interests include power industry restructuring, power system alarm processing, fault diagnosis and restoration strategies, as well as smart grids and electric vehicles.

Prof. Wen is an associate editor of IET Generation, Transmission and Distribution, ASCE's Journal of Energy Engineering, and Springer's Journal of Modern Power Systems and Clean Energy. 